\title{
New Developments in Proton Radiography at the Los Alamos Neutron Science Center (LANSCE)
}

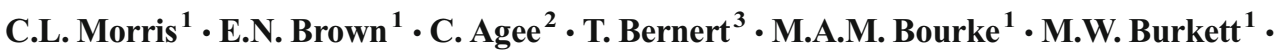 \\ W.T. Buttler ${ }^{1}$ - D.D. Byler ${ }^{1}$ - C.F. Chen ${ }^{1}$ - A.J. Clarke ${ }^{1}$. J.C. Cooley ${ }^{1}$ P.J. Gibbs ${ }^{1}$ • \\ S.D. Imhoff ${ }^{1} \cdot$ R. Jones $^{2} \cdot$ K. Kwiatkowski ${ }^{1}$ - F.G. Mariam ${ }^{1}$ • F.E. Merrill ${ }^{1}$ \\ M.M. Murray ${ }^{1}$ - C.T. Olinger ${ }^{1}$ - D.M. Oro ${ }^{1} \cdot$ P. Nedrow $^{1} \cdot$ A. Saunders ${ }^{1} \cdot$ G. Terrones $^{1}$ •

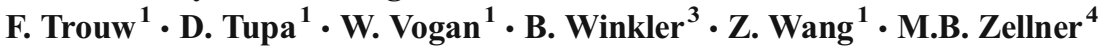

Received: 10 October 2014 / Accepted: 16 July 2015 / Published online: 30 December 2015

(C) The Author(s) 2015. This article is published with open access at Springerlink.com

\begin{abstract}
An application of nuclear physics, a facility for using protons for flash radiography, has been developed at the Los Alamos Neutron Science Center (LANSCE). Protons have proven far superior to high energy $\mathrm{x}$-rays for flash radiography because of their long mean free path, good position resolution, and low scatter background. Although this facility is primarily used for studying very fast phenomena such as high explosive driven experiments, it is finding increasing application to other fields, such as tomography of static objects, phase changes in materials and the dynamics of chemical reactions. The advantages of protons are discussed, data from some recent experiments will be reviewed and concepts for new techniques are introduced.
\end{abstract}

Keywords Proton radiography $\cdot$ Dynamic materials $\cdot$ Static applications · Tomography · Detonation propagation · Richtmyer-Meshov instability · Metal jets · Casting $\cdot$ Fuel rods $\cdot$ Chelyabinsk $\cdot$ Meteorite

C. L. Morris

cmorris@lanl.gov

1 Los Alamos National Laboratory, Los Alamos, NM 87544, USA

2 University of New Mexico, Albuquerque, NM, USA

3 Goethe-Universität Frankfurt am Main, Institut für Geowissenschaften, Abt. Kristallographie, Altenhöferallee 1, 60438 Frankfurt am Main, Germany

4 U.S. Army Research Laboratory, RDRL-WMP-D, Aberdeen, MD 21005, USA

\section{Introduction}

In the mid-1990's a new tool to aid in the mission of stewarding the US nuclear stockpile was invented at Los Alamos, namely proton radiography (pRad) [1]. The concept uses the attenuation due to the nuclear scattering of very short pulses of energetic protons as they transit high explosive driven experiments to provide contrast for flash radiography. The long mean free path of intermediate energy (10's of GeV) protons mitigates many of the difficulties encountered over the previous 5 decades of flash radiograph with high energy $\mathrm{X}$-rays, such as large scatter backgrounds, low dynamic range, and poor position and temporal resolution [2, 3].

Experiments preformed using $24 \mathrm{GeV}$ protons provided by the Alternating Gradient Synchrotron (AGS) accelerator at Brookhaven Nation laboratory demonstrated all of the expected gains from pRad when compared to X-rays with a set of unclassified [4] and classified static objects [5]. The use of pRad for dynamic testing of surrogate nuclear weapon primaries is being pursued by both the Russian [3, 6] and Chinese [7-11] weapons program but not by the United States.

However, a proton flash-radiography facility that uses the $800 \mathrm{MeV}$ beam from the Los Alamos Neutron Science Center (LANSCE) at Los Alamos National Laboratory has become a work horse for the US stockpile stewardship program for smaller experiments studying the science of explosively driven systems. A recent review describes the techniques of proton radiography and presents some experimental results [2].

In this paper we briefly describe the technique, examine some of the current capabilities of the LANSCE proton radiography facility, illustrate them with some resent results, and discuss possibilities for the future. 


\section{Proton Radiography}

The interaction of energetic protons with matter is governed to high precision by nuclear and Coulomb interactions. Protons lose energy to the matter because of the Coulomb scattering of the protons from the atomic electrons, and they scatter from the nuclei both because of the strong interaction and the Coulomb interaction with the proton. For the purposes of understanding proton radiography these interactions can be factorized and treated independently. The energy loss is given by the BetheBloch model [12], Coulomb scattering is given by the Moliere theory $[13,14]$, and nuclear scattering can be described by the black disk or optical model of nuclear scattering [15].

Proton radiography is performed by illuminating a target object with a beam of protons and then by focusing the transmitted protons onto a scintillator screen $[16,17]$ using a quadrupole magnetic lens $[18,19]$. A collimator located at the Fourier plane of the lens is used to control the amount of contrast produced by Coulomb scattering. Nuclear interactions scatter or absorb beam particles generally to angles far outside the multiple scattering cone, which is on the order of $10 \mathrm{mrad}$ in typical LANSCE experiments. The formalism used for analyzing pRad data is described in reference [2] and a summary of the physics listed above can be found in the particle data review [20].

The transmission, $t$, through an object of thickness, $z$, for proton radiography through a lens with a collimator acceptance angle, $\theta_{C}$, is the product of the nuclear attenuation and the Coulomb attenuation, the proton radiography transmission equation [2]:

$t=e^{-\frac{z}{\lambda}}\left(1-e^{-\frac{\kappa}{z}}\right)$

where :

$\kappa=\frac{\theta_{c}^{2} p^{2} \beta^{2} X_{0}}{(14.1 \mathrm{MeV})^{2}}$

and :

$\lambda=\frac{1}{\rho \sigma_{A}}$

The absorption cross section, $\sigma_{A}$, for hadrons on a nucleus with mass number A is often approximated by $\sigma_{A}=\pi r_{A}^{2}$ (accurate to $\approx 20 \%$ ), the geometric cross section of the nucleus, where $r_{A} \approx 1.2 A^{\frac{1}{3}} \mathrm{fm}$. Here $p$ is the proton momentum, $\beta$ is its velocity relative to the speed of light, and $X_{0}$ is the radiation length [21]. For monolithic materials the proton radiography transmission equation can be inverted to obtain the thickness of an object. For objects where $z<<\lambda$, one can obtain radiation weighted thicknesses by solving equation (1). Because of generally small backgrounds precise thicknesses (on the order of one percent) can be obtained from proton radiography in these cases [22].

\section{LANSCE Capabilities}

The radiography facility at LANSCE is located in the former High Hesolution Spectrometry beam area (Experimental Area C) of Los Alamos Meson Physics Facility, LAMPF. It consists of an achromatic beam transport line into the area, a diffuser and matching section to control the size of the incident beam and reduce chromatic aberrations, a set of proton lenses, a containment vessel for dynamic experiments, and an optical imaging system. The LANSCE accelerator produces an $800 \mathrm{MeV} \mathrm{H}$ - proton beam of macro pulses with widths of up to $1 \mathrm{~ms}$ and a spacing of $8.33 \mathrm{~ms}(120 \mathrm{~Hz})$.

Each macro pulse consist of a string of micro pulses spaced by $5 \mathrm{~ns}$ with a widths of $100 \mathrm{ps}$. The time control system for the accelerator allows a set of pulses to be constructed from any combination of micropulses within a macropulse for proton imaging.

The protons are delivered though a beam line that consists of a set of phosphor screens viewed by CCD cameras for beam monitoring and alignment, a strip line detector for measuring the time structure of the beam, a fast transformer for measuring the beam pulse intensity, a set of upstream quadrupole magnets for adjusting the angle position correlation of the beam on the sample to control chromatic aberrations, and a mechanical assembly that can insert any one of a set of tantalum diffusers to control beam spot size on the target.

Three lenses with different magnification can be used for radiography with magnifications of $\times 1$ [19], $\times 3$ [18], and $\times 7$ [23]. The $\times 1$ lens is constructed from four $30 \mathrm{~cm}$ diameter bore, $60 \mathrm{~cm}$ long quadrupole electromagnets and the $\times 3$ from six $10 \mathrm{~cm}$ diameter bore $20 \mathrm{~cm}$ long permanent quadrupole magnets. The magnifying lenses $(\times 3$ and $\times 7)$ use Neodymium Iron Boron quadrupole magnets that need to be periodically remagnetized to repair radiation induced changes in the remnant magnetic field [24]. The $\times 7$ lens is being upgraded to use Samarium Cobalt magnets to mitigate this problem.

We have measured the spatial resolution of the three lens systems at LANSCE by placing $3 \mathrm{~mm}$ thick targets of tungsten at the object location and characterizing the edge width. (The thickness of the target can lead to alignment issues so the data are not entirely consistent from year to year). Some results are shown in Fig. 1, along with estimates of the resolution made by fitting the edge with an error function. The resolution is given as the width.

There are many contributions that limit the resolution. These include chromatic aberrations in the magnetic lens system, proton scattering in the exit window and radiation to light converter. Of these, all but the last decrease approximately as $1 / \mathrm{M}$ where $\mathrm{M}$ is the magnification of the lens system. The spatial resolution measurements, displayed in Fig. 1 are plotted in Fig. 11 vs. 1/M. These data agree well with a linear dependence on 1/M Fig. 2. 
Fig. 1 Spatial resolution measurements for the existing LANSCE proton radiography lens systems. Images of an edge for each of the three lenses are shown in the top row and plots of transmission vs. position along the yellow line are shown in the bottom graphs. The spatial resolution $(\Delta \mathrm{x})$ extracted by fitting an error function to the transmission vs. position are shown in the text boxes

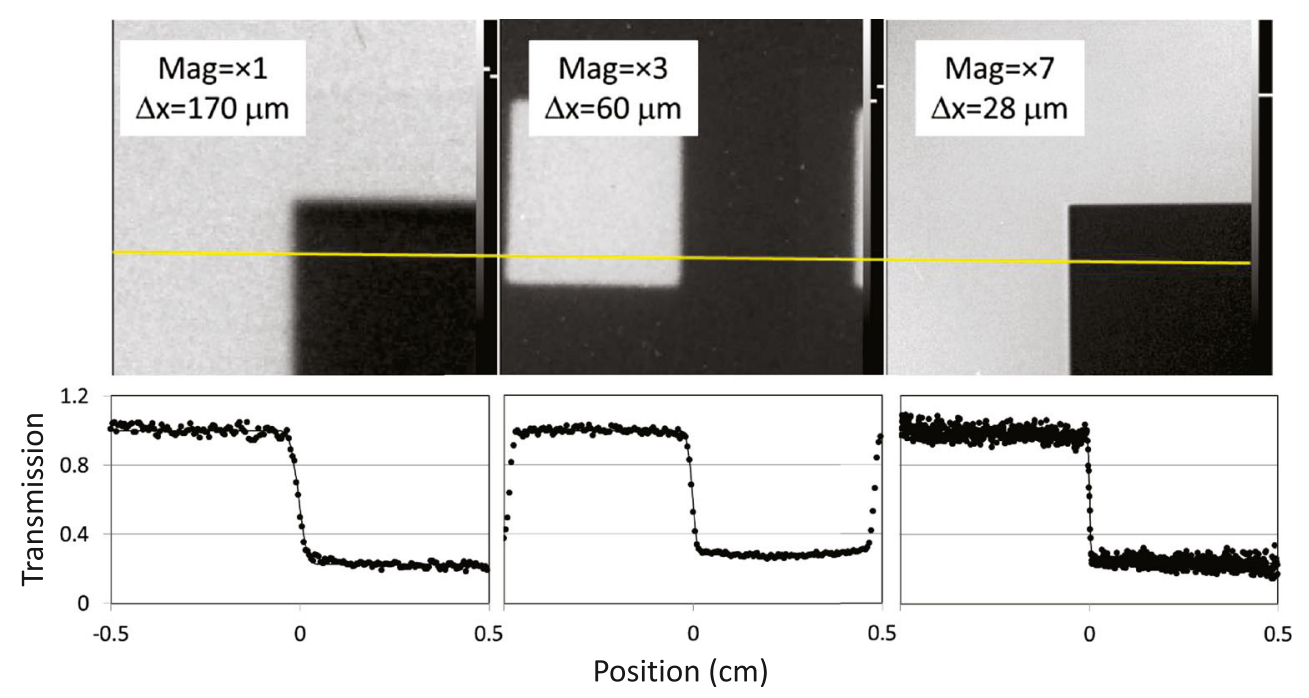

\section{Dynamic Experiments}

Proton radiography has enabled new classes of experiments that are not effectively performed with flash x-rays. These have included studies of detonation propagation in high explosives, armor penetration experiments, and instability growth in shocked and accelerated interfaces. Here we present a few examples.

\section{Detonation Propagation and Reflected Shock Interactions with the $\times 1$ Lens}

Proton radiography has been used to investigate the spatiotemporal evolution of detonation fronts and the associated reflected shocks on a PBX-9502 high explosive charge between an outer cylindrical steel liner and an inner elliptical tin liner (shown in Fig. 3) [25, 26]. The charge was initiated with a PBX-9501 booster and a line wave generator (LWG) at $30^{\circ}$ from the major axis of the ellipse. This configuration provided a large region where the high explosive is not within the line of sight of the detonation line and thus the data can be used to test various burn models and equation of state formulations. A single frame of the 21 frame movie is shown in Fig. 3.

\section{Richtmyer-Meshkov Instability with the $\times 3$ Lens}

A number of experiments have been performed to study the spike and bubble growth in plane wave driven Richtmyer-Meshkov instability growth in both solid and liquid metals [27-31]. These data have been used to develop new models of the dynamics of instability growth and ejecta formation with unsupported shocks. A very recent development is a two wave driver for studying "second shock" effects on instability growth [27]. Proton radiography data taken of this system will allow for a more detailed quantitative understanding of two-shock driven instability and the resulting ejecta formation. Some initial data from Buttler et al. [27] are shown in Fig. 4.

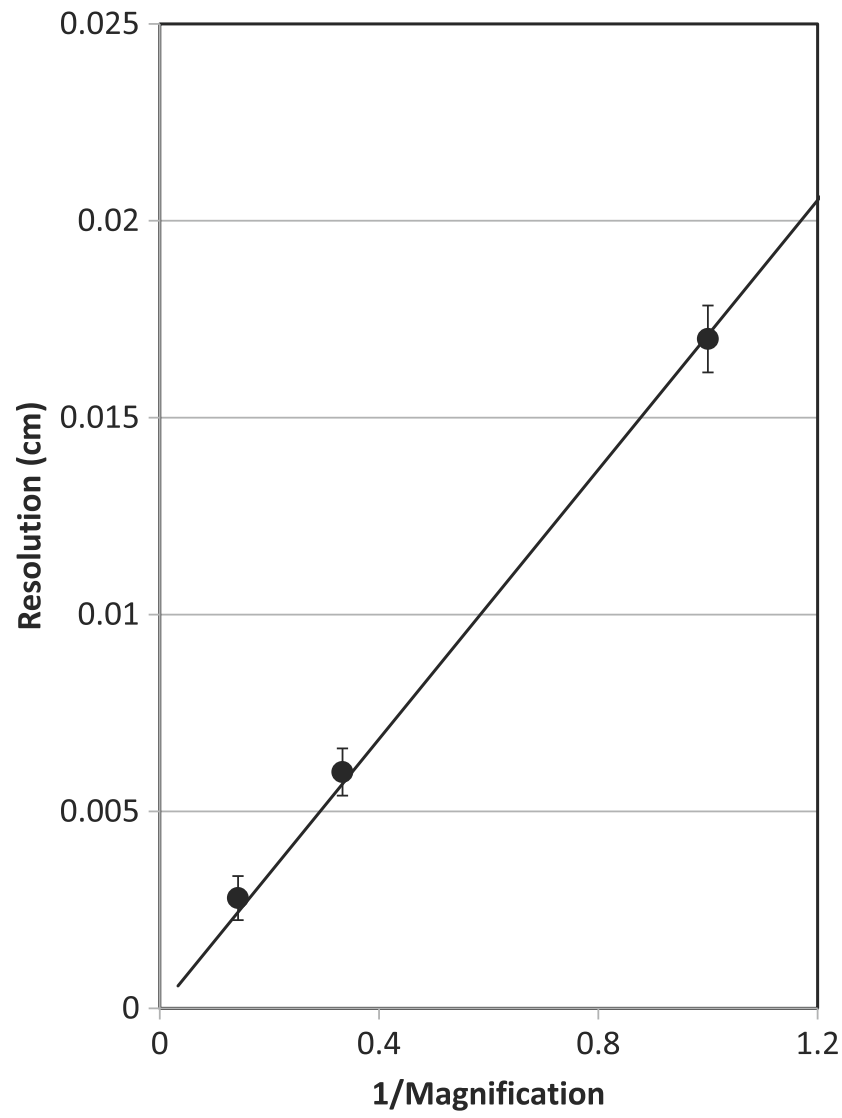

Fig. 2 Measured position resolution as a function of inverse magnification. The line shows a linear fit to the data 
Fig. 3 Left photo of the experiment, Right one frame of data taken at $13.8 \mu$ s showing the burn front and reflected shock
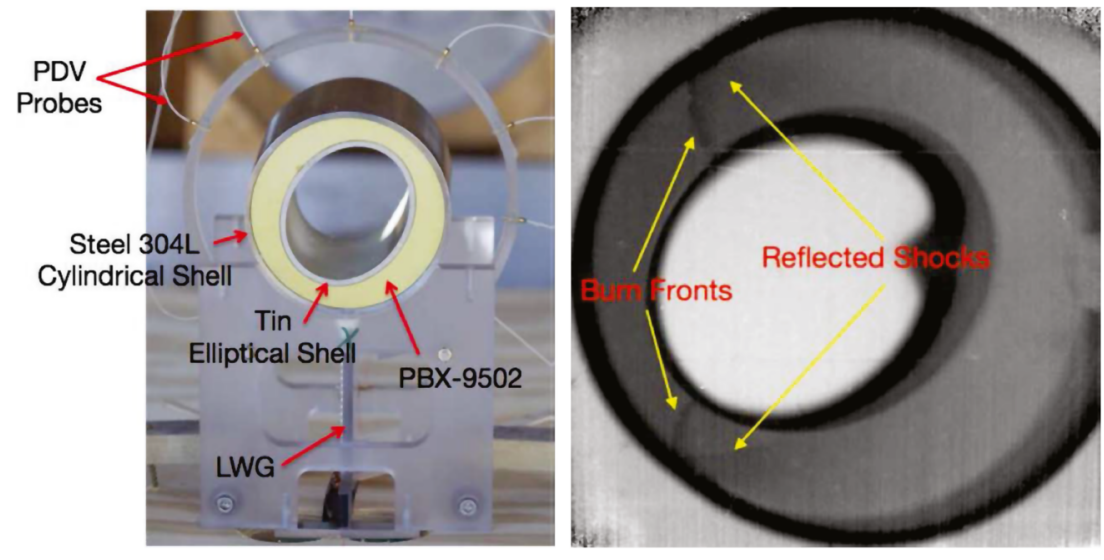

\section{Metal Jets with the $\times 1$ Lens}

Another activity where proton radiography is advancing our understanding of dynamic phenomena is in the field of armor development. A large number of experiments have been performed to study the disruption of metal jets by various armor configurations and materials. An example of early-time data is shown in Fig. 5, where a metal jet is penetrating a cylinder of glass.

\section{Quasi-Static Experiments}

The penetration, resolution, flexible time format, and long standoff make proton radiography useful for a number of static or quasi-static applications. Some examples demonstrating the breadth of this work are given in the following subsections.

\section{Casting Experiment with the $\times 1$ Lens}

The $\times 1$ lens provides a $12 \times 12 \mathrm{~cm}^{2}$ field of view useful for imaging large scale phenomena. Clarke et al. [32] and Gibbs et al. [33] have reported data spanning the micro-scale to the mesoscale (obtained with proton radiography and synchrotron $\mathrm{X}$-ray radiography) and the macroscopic scale (obtained with proton radiography). Such data can be used to study metal solidification dynamics across length scales to validate and improve models of important manufacturing processes such as casting.

As an example at the macroscopic scale, a tin-bismuth alloy melt flowing into a $3 \mathrm{~mm}$ thick graphite mold is shown in Fig. 6. Here darker regions correspond to higher densities. The fill process was controlled by a plug between the reservoir and the mold made of pure bismuth which had a higher melting temperature than the alloy melt. As the reservoir was heated and the plug melted, the fill was initiated. Proton images were made using a $350 \mu \mathrm{m}$ thick columnar $\mathrm{CsI}(\mathrm{Tl})$ screen, and recoded on a DIMAX CMOS $2 \mathrm{k} \times 2 \mathrm{k}$ pixel [34] camera. Each frame was formed with a beam pulse of $\sim 6 \times 10^{9}$ protons, distributed as a two dimensional Gaussian across the $12 \times 12 \mathrm{~cm}^{2}$ field of view at the object location.

When the temperatures approached the melting temperature of the plug, a 1000 frame movie was started at $5 \mathrm{~Hz}$. The frames below represent about $3 \mathrm{~s}$ of the $200 \mathrm{~s}$ movie that covered the filling of the mold. The flexible timing of the linear accelerator for proton radiography was useful to enable capture of the region of interest, without the complication of a trigger to synchronize the beam to the experiment.

\section{Radiography of Surrogate Fuel Rods with the $\times 3$ Lens}

There are several advantages to proton radiography when compared to other radiographic probes. One is the long standoff between the object and the image location. This has obvious advantages for explosively driven experiments in which it is necessary to protect
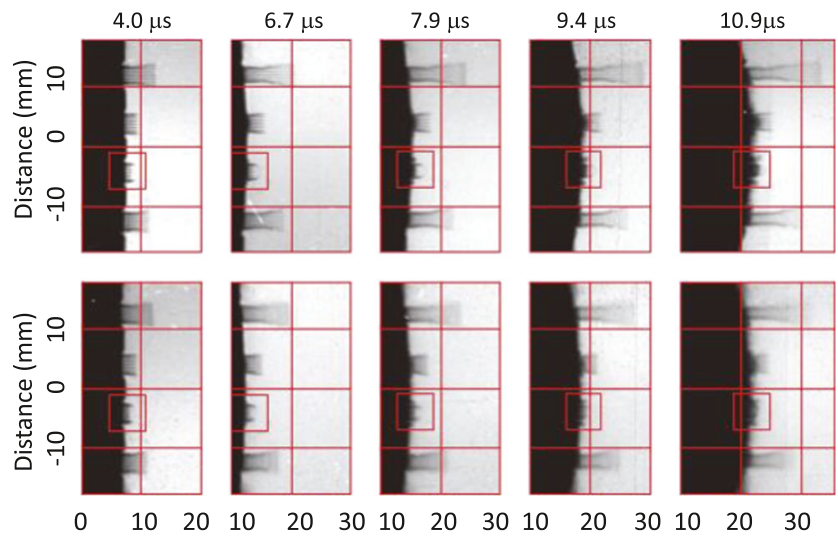

$$
\text { Distance }(\mathrm{mm})
$$

Fig. 4 pRad radiographs of twice shocked tin targets that included large scale perturbations. The top images show instabilities driven into vacuum and the bottom images are into about 14 psia neon gas 
Fig. 5 Movie of a metallic jet penetrating into a cylindricly shaped armor material. Time increases from the left right image
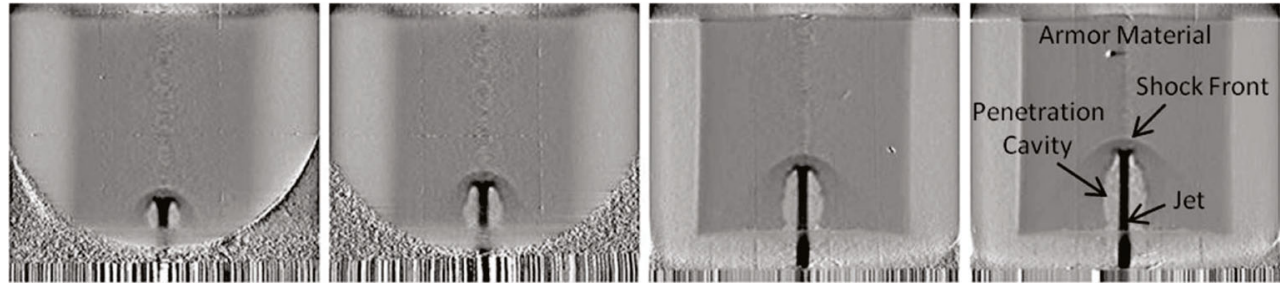

the detector from explosive products. Another case is in radiography of highly radioactive targets.

Considerable effort is aimed at developing models that can predict structural changes to nuclear fuel pellets as a function of burnup in a reactor. Extending the lifetime has obvious economic benefits. Proton radiography provides data that can provide $<50 \mu \mathrm{m}$ position resolution for objects of the scale of $5 \mathrm{~cm}$, with areal densities of $10 \mathrm{~g} / \mathrm{cm}^{2}$ of uranium oxide or thorium oxide, and in a radiation field that can be larger than $200 \mathrm{~Gy} / \mathrm{h}$ at $30 \mathrm{~cm}$ from the fuel rod, because of its short exposure times and long standoff (the cameras and scintillator are $12 \mathrm{~m}$ from the object location).

Here we show data taken on a set of objects with the $\times 3$ magnifier aimed at determining the capabilities of proton radiography for evaluating the damage of activated fuel rods. A previous subset of this data were used to compare X-ray and proton radiography [35] and to demonstrate the isotopic sensitivity of resonance neutron imaging [36]. Plausible methods for using these other probes in the radiation fields of near the activated fuel rods have not been described. With protons the long standoff and fast imaging provide a solution to this problem.

Tomographic proton data were taken on several surrogate fuel assemblies. Proton images were made using a $350 \mu \mathrm{m}$ thick columnar $\mathrm{CsI}(\mathrm{Tl})$ screen, and recoded on a set of 5 three frame CMOS imaging cameras [37].
Seven hundred and twenty frames of data were taken covering $180^{\circ}$ by rotating the object by 0.25 degrees between frames. Each frame was formed from an average of 15 camera pictures taken using 3 beam pulses of $6 \times 10^{9}$ protons distributed as a two dimensional Gaussian across the $4 \times 4 \mathrm{~cm}^{2}$ field of view at the object location with a widths of $1.3 \mathrm{~cm}$ in each direction. The proton data were taken at a rate of $0.5 \mathrm{~Hz}$. The rate was limited by the speed at which the goniometer could reliably rotate the object.

The proton radiography equation was inverted to obtain areal densities. Volume densities were calculated using filtered back projection. In Fig. 7 the results obtained from four different test objects are shown: a set of $10 \mathrm{~mm}$ diameter thoria pellets, a set of $5 \mathrm{~mm}$ diameter urania fuel pellets compressed to at a range of densities, and two sets of $5 \mathrm{~mm}$ diameter urania pellets with various defects emplaced before the sintering process.

The ensemble of data demonstrates a range capability: the ability to quantitatively measure density, Fig. 7(c); the observation of texture in the thick thoria pellets Fig. 7(a); the wide dynamic range of protons in thickness; and the sensitivity of protons to $\sim 60 \mu \mathrm{m}$ features Fig. 7(b) and (d). The texture in the thoria was first characterized in the proton radiography results. The density determinations of the pellets in Fig. 7(c) were within a few percent of the measured densities of the

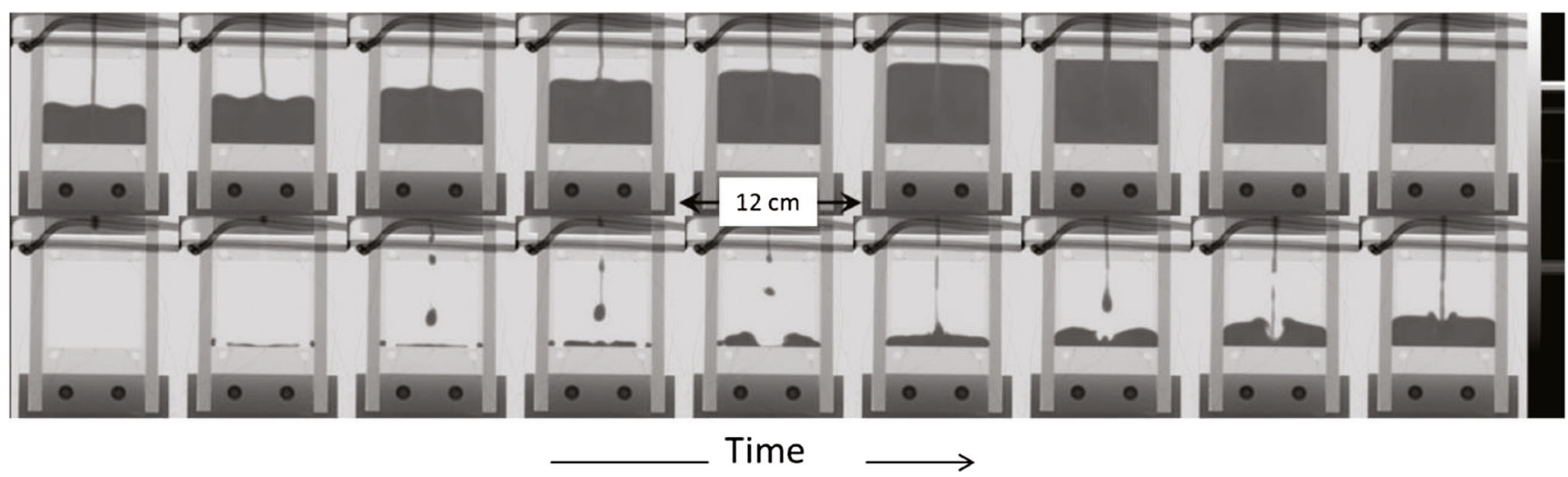

Fig. 6 Casting mold filling of a 3-mm-thick plate using the $\times 1$ lens (tin 27 atomic \% bismuth alloy). Darker regions correspond to higher density. Spacing between frames is $0.2 \mathrm{~s}$ 


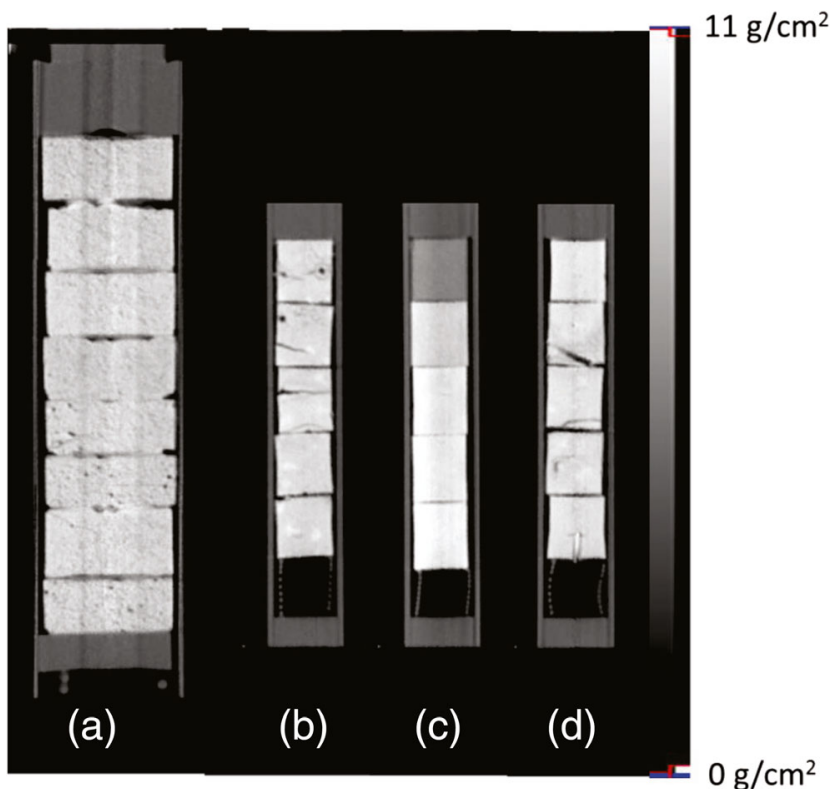

Fig. 7 Center slices from the fuel pellet tomographs. A scale bar for the densities is shown on the right

sintered samples. The urania samples were interesting because the proton data could be used to observe the migration and changes that occurred in the embedded defects during the sintering.

\section{Radiography of Self-Propagating High Temperature Chemical Reactions with the $\times 3$ Lens}

The $120 \mathrm{~Hz}$ macro structure of the LANSCE accelerator allows continuous radiography of experiments with dynamic structure, but where the initiation time is difficult to predict. Here we present an example of a movie of a self-propagating high temperature reaction [38-45] in a stoichiometric mixture of titanium and silicon. In these reactions the material changes from a pressed powder to a ceramic pellet. Proton radiography allows study of the dynamics, perhaps aimed at process control for producing near net shaped ceramic parts dynamically.

Data from one test from Bernert et al. [46] are shown in Fig. 8. Figure 8(a) shows a frame just after initiation. Near the top, the tungsten filament that was used to initiate the reaction can be observed. The sequence of radiographs, shown in Fig. 8b, was cut out of a movie that was approximately $100 \mathrm{~s}$ long. This allowed the collection of dynamic data even through the initiation time was quite uncertain. The data were taken with the $\times 3$ magnifier, with a position resolution of $\sim 50 \mu \mathrm{m}$. The exposure times were $\sim 180 \mathrm{~ns}$, ensuring there is negligible motion blur. The frame rate was $10 \mathrm{~Hz}$. The data allowed precise measurements of the location and shape of the reaction zone shown in Fig. 8(b) as a function of time and quantitative measurements of the time dependence of the density, Fig. 8(c). The average flame front velocity was determined to be $0.7 \mathrm{~cm} / \mathrm{s}$.

\section{Tomography of a Meteorite Sample with the $\times 7$ Lens}

The $\times 7$ magnifier proved to be difficult to commission. Although it worked well in a commissioning run, it failed to perform as expected in later runs. Work with the $\times 3$ magnifier has definitively demonstrated that radiation damage leads to demagnetization of the permanent magnet material, particularly in regions of high dose and large differences between the field direction and the magnetization directions [24]. This leads to non-uniform reduction in field strength, which only weakens the focusing slightly, but introduces large optical aberrations in the lens, which results in significant resolution degradation. This problem is especially difficult with the $\times 7$ magnifier because of the small bore which places the neodymium iron boron very close to the intense proton beam.

With this knowledge the $\times 7$ magnifier was recently recommissioned after remagnetization and it was used to perform several of experiments. One of these was tomography on a small sample of the meteorite the exploded over Chelyabinsk Russia in 2013.

After tuning the lens and performing some of experiments that needed only a few radiographs, the meteorite sample was radiographed at 721 angles spanning $360^{\circ}$. The degradation of the resolution across this set of radiographs is apparent in Fig. 9.

Two representative slices from the tomograph are shown in Fig. 10. One can clearly observe higher- $Z$ regions that are likely to be metal inclusions in a silicate base. Many of the small inclusions seem to have a low density region at their center. The line plot shows one of these features.

A new design has been completed for this lens that has shown that the neodymium iron boron magnets can be replaced with much more radiation resistant samarium-cobalt magnets. The new system has been procured and will be commissioned over the next year.

\section{Scanning Proton Microscope}

Two new ideas can enable major advances in proton radiography for the sort of quasi-static experiments described above. The first is the realization that by scanning a pencil beam the limiting position resolution in proton radiography is determined by the size of the incident beam and scattering in the target, which can be much smaller than the resolution obtained with lens focusing because the energy and angle spread leading to chromatic blur are no longer an issue. 

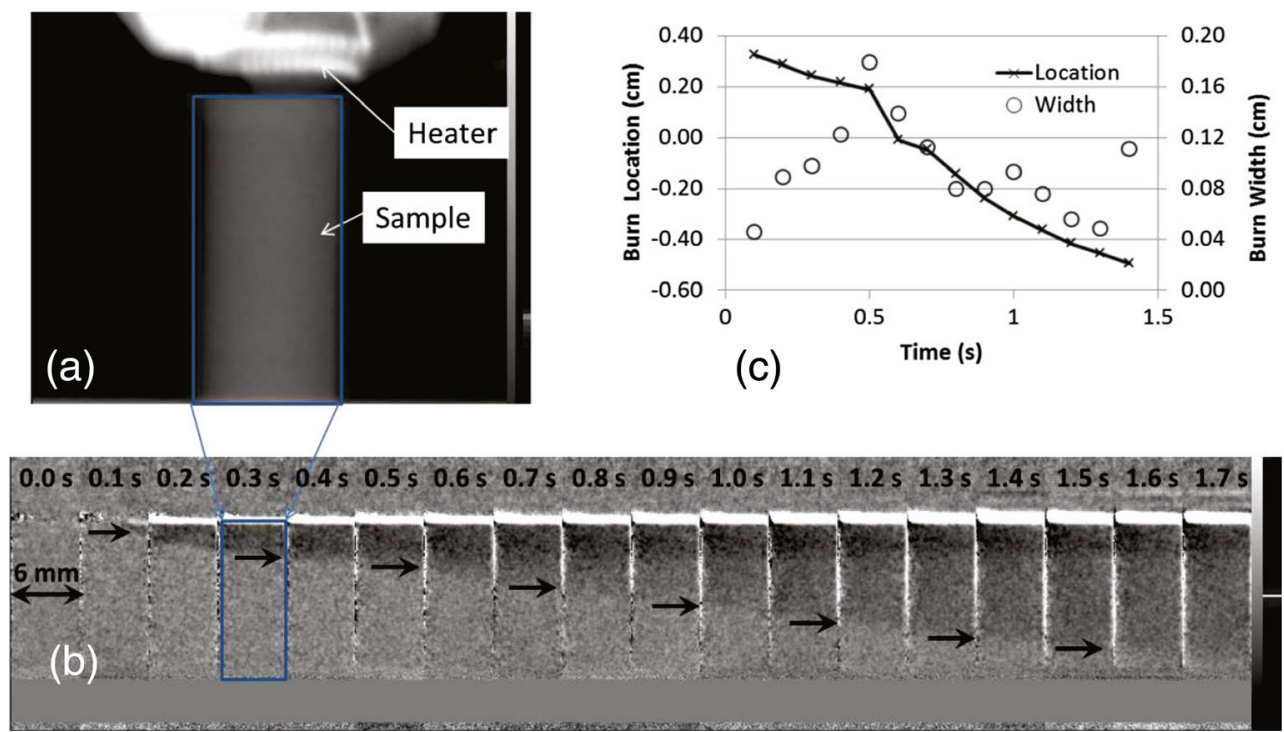

Fig. 8 (a) Areal densities calculated from a radiograph of an early frame of a radiographic movie showing the thermal induced chemical reaction $5 \mathrm{Ti}+3 \mathrm{Si} \rightarrow \mathrm{Ti}_{5} \mathrm{Si}_{3}$. (b) Time dependent measurements of the location and shape of the reaction zone. Arrows mark the location of the reaction zone. Images showing the ratios of the areal density to an average of

When protons traverse material there is diffusion of angles and positions, as shown in Fig. 11. For a target of thickness $t$, the blur from scattering in the target is [21]

$\Delta y=\frac{\theta_{0} t}{\sqrt{12}}$, where $\theta_{0}=\frac{1}{p \beta} \sqrt{\frac{t}{X_{0}}}$

As an example, for a $3 \mathrm{~mm}$ thick sample of uranium the above gives $\Delta \mathrm{y}=5 \mu \mathrm{m}$ with an $800 \mathrm{MeV}$ proton beam. This is much better resolution than lens focused radiography can provide. With a collimated beam, a measurement of transmission though an angle collimator measures the object thickness. An object can be radiographed by measuring the attenuation as a function of position with either a scanned beam or a scanned object. A collimated beam can be produced by imaging the beam transmitted through a pinhole. A further reduction in the beam size can be obtained by using a demagnifying lens to focus the beam onto the object.

The beam from the LANSCE accelerator is produced with $201 \mathrm{MHz}$ microstructure. Individual beam pulses are about 100 ps long spaced by $5 \mathrm{~ns}$. New developments in both detectors and recording technology make $10 \mathrm{ps}$ measurements now possible. By using a fast detector one can measure the time of flight of the protons through the system and determine their energy as well as the intensity. Thus one can determine the energy loss of protons in a target. The energy loss, $\frac{d E}{d y}$, of protons traversing material is given by the Bethe-Bloch formula $[12,21]$. The energy loss only varies slightly across through the periodic table from $2 \mathrm{MeV} /\left(\mathrm{g} / \mathrm{cm}^{2}\right)$ in low-Z targets to $1.5 \mathrm{MeV} / \mathrm{g} / \mathrm{cm}^{2}$ in high-Z targets at LANCE proton several radiographs made before initiation of the reaction from unreacted (left) to fully reacted (right). (c) The result from fits to the reaction zone showing the location and width as the reaction proceed. $5 \mathrm{Ti}+3 \mathrm{Si} \rightarrow \mathrm{Ti}_{5} \mathrm{Si}_{3}$

energies. The energy of transmitted protons can be determined by their time of flight through the lens. One finds that the energy loss, $\Delta E$, is related to the change in velocity $\Delta \beta$ by: $\Delta E=\Delta \beta \beta \gamma^{2} E$, where $\mathrm{E}$ is the total energy, $\beta$ is the velocity relative to the speed of light, and $\gamma=1 / \sqrt{1-\beta^{2}}$.

With $10^{4}$ transmitted protons in each 100 ps long proton pulse, it is possible to obtain time resolution, $\Delta t=100 \mathrm{ps} / \sqrt{10^{4} \text { protons }}=1 \mathrm{ps}$, when the measurement is limited by proton counting statistics. A $24 \mathrm{~m}$ flight path gives sufficient energy resolution to measure the thickness of the $3 \mathrm{~mm}$ thick uranium sample to about $0.5 \%$, about the same precision obtained with multiple scattering radiography with the current system. Combining energy loss information:

$$
\frac{d E}{d x}=K z^{2} \frac{Z}{A} \frac{1}{\beta^{2}}\left[\frac{1}{2} \ln \left(\frac{2 m_{e} c^{2} \beta^{2} \gamma^{2} T_{\max }}{I^{2}}-\beta^{2}-\frac{\delta(\beta \gamma)}{2}\right],\right.
$$

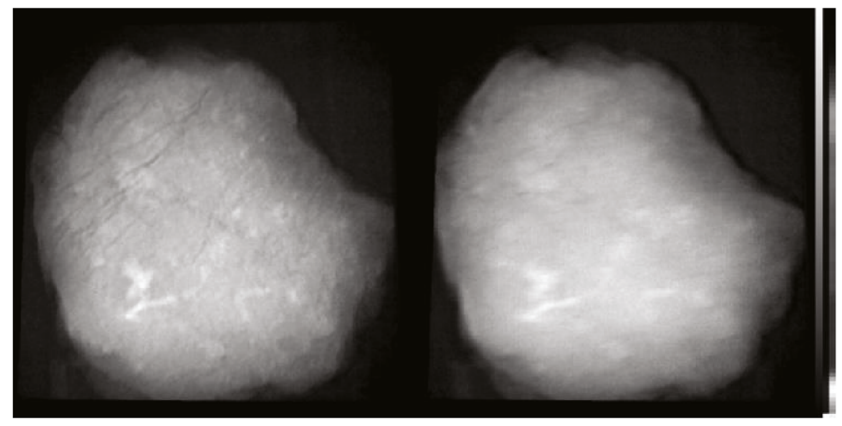

Fig. 9 Areal density measured at the start and finish of the 721 frame sequences shown the degradation of the spatial resolution caused by radiation damage to the lens magnets 
Fig. 10 Representative slices from the tomographic reconstructions. X-y slices showing the volume density are shown above line plots of the density corresponding to the white lines. An assortment of features can be observed throughout the sample. The bright patches, marked with an arrow on the left, are interpreted to be higher- $\mathrm{Z}$ inclusions and on the right a smaller one that appears to have some structure
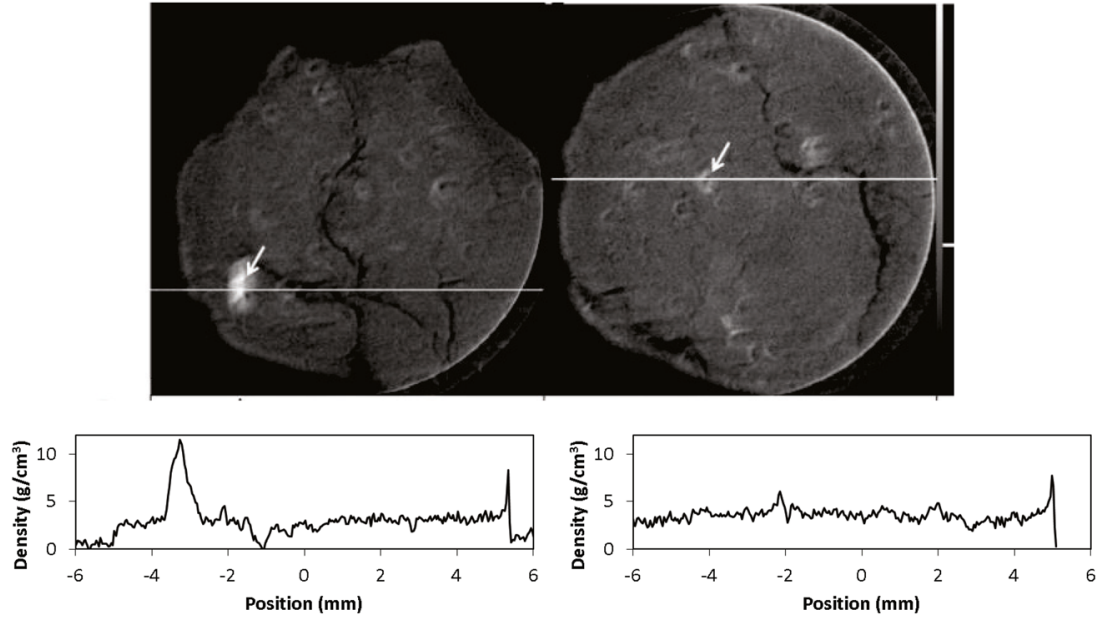

with multiple scattering information which has an extra power $\mathrm{Z}$ when compared to energy loss one can determine thickness and the mean atomic number of the material being radiographed. The key items for determining time of flight (TOF) to this precision are fast transparent beam monitors, fast transmitted beam detectors and fast recording.

\section{Preliminary TOF Radiography Studies}

We have taken some preliminary data using a $20 \mathrm{GHz}$ analog bandwidth oscilloscope and a fast multi-channel plate detector. The beam was collimated to a root mean squared spot size of $\sim 150 \mu \mathrm{m}$ for these measurements.

TOF data between an upstream strip line detector and the downstream channel plate detector are shown in Fig. 12. Five measurements were made with trains of 80 micropulses spaced by 5 ns with $10^{5}$ protons per micropulse. Time drifts on the order of $100 \mathrm{ps}$ (corresponding to about $5 \mathrm{MeV}$ of

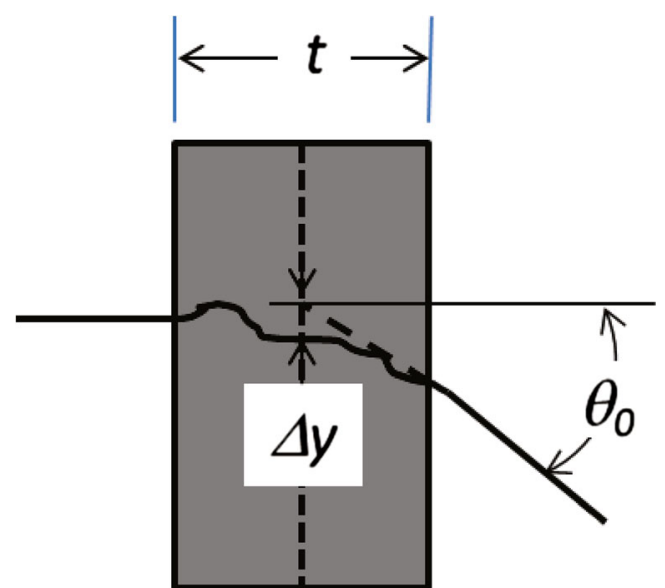

Fig. 11 Quantities used to describe Coulomb multiple scattering in the plane of the figure energy shift) both across the pulse train and between pulses are evident. The beam energy shifted within a macro-pulse and from pulse to pulse. These shifts are likely to be due to changes in the beam energy because of RF loading in the linera accelerator (LINAC). In future measurements the beam energy can be measured by comparing the outputs from two upstream detectors separated by $>10 \mathrm{~m}$ to measure the beam energy and correct for these shifts.

\section{Summary}

An overview of the LANCE proton radiography system and the experimental program has been given. The position resolution of the $\times 1, \times 3$, and the $\times 7$, lenses has been measured and found to depend linearly on inverse magnification over this range of magnifications. This suggests that that further improvements can be obtained at increased magnification. Design studies of higher magnification systems are underway.

Examples of static or quasi-static experiments have been presented for each of the lenses. These experiments cover a

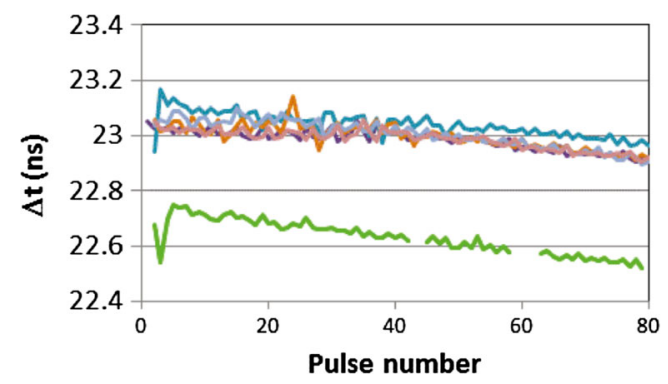

Fig. 12 Proton time measurements for $400 \mathrm{~ns}$ long trains of $5 \mathrm{~ns}$ spaced pulses. The upper data sets are repeat measurements of the flight time between an upstream strip line detector and a downstream multi-channel plate detector. The green curve shows the effect of adding $1.25 \mathrm{~cm}$ of aluminum causing about $5 \mathrm{MeV}$ of energy loss to the proton beam 
wide sampling of applications and suggest that as the capabilities of $\mathrm{pRad}$ improve, the number of applications for basic science and engineering will grow, especially in areas where the standoff, wide dynamic range and long pulse sequences and flexible time format provided by proton radiography are an advantage.

The possibility of improving the position resolution while providing material identification by using pencil beams and by measuring time of flight as well as attenuation has been described. The addition of these new measurement capabilities would significantly increase the utility of proton radiography for both quasi-static and some dynamic experiments.

Acknowledgments This work was performed under the auspices of the U.S. Department of Energy under Contract DE-AC5206NA25396. This work benefited from important contributions from the LANSCE pRad team and accelerator staff. We gratefully acknowledge the support of the U.S. Department of Energy (DOE) through the LANL/LDRD Program for this work. A. J. C., S. D. I., P. J. G. and the casting mold filling experiment were supported by A. J. C.'s Early Career award from the U.S. DOE, Office of Basic Energy Sciences, Division of Materials Sciences and Engineering.

Open Access This article is distributed under the terms of the Creative Commons Attribution 4.0 International License (http:// creativecommons.org/licenses/by/4.0/), which permits unrestricted use, distribution, and reproduction in any medium, provided you give appropriate credit to the original author(s) and the source, provide a link to the Creative Commons license, and indicate if changes were made.

\section{References}

1. Gavron A, Morris CL, Ziock HJ, Zumbro JD (1996) Proton Radiography, Los Alamos National Laboratory Report No. LAUR-96-420. Los Alamos National Laboratory, Los Alamos

2. Morris C, King NS, Kwiatkowski K, Mariam FG, Merrill FE, Saunders A (2013) Charged particle radiography. Reports on progress in physics. Phys Soc (Great Brit) 76(4):046301

3. Antipov YM, Afonin AG, Vasilevskii AV, Gusev IA et al (2010) A radiographic facility for the $70-\mathrm{GeV}$ proton accelerator of the institute for high energy physics. Instrum Exp Tech 53(3):319-326

4. Morris CL, Ables E, Alrick KR, Aufderheide MB et al (2011) Flash radiography with $24 \mathrm{GeV} / \mathrm{c}$ protons. J Appl Phys 109:104905

5. Morris CL, Alrick KR, Buescher KL, Cagliostro DJ et al (2004) Comparison of proton \& $\mathrm{x}$-ray thick object radiography (U). Def Res Rev 11(3):51-57

6. Kolesnikov SA, Golubev AA, Demidov VS, Dudin SV et al (2010) Application of charged particle beams of TWAC-ITEP accelerator for diagnostics of high dynamic pressure processes. High Press Res 30(1):83-87

7. He X, Yang G, Liu C (2008) Optimization research on image lens of proton radiography. High Power Laser Part Beams 20(2):297300

8. Liu J, Zhang L, Liu J, Shi J-j (2009) Diffuser design for FTO's proton radiography. High Power Laser Part Beams 21(3):455-458

9. Liu J, Zhang L, Shi J, Liu J (2010) Secondary proton removing effects of imaging lenses in proton radiography. Qiangiiguang Yu Lizishu/High Power Laser Part Beams 22(12):3031-3033
10. Liu J, Zhang L-w, Liu J, Shi J-j (2008) Blur caused by container in proton radiography. High Power Laser Part Beams 20(7):11991202

11. Yang G-j, Zhang Z, Wei T, He X-Z, Long J-D, Shi J-S, Kai-Zhi Z (2012) A design study of a magnifying manetic lens for proton radiography. Chin Phys C 36(3):247-250

12. Bethe $H$ (1930) Theory of passage of swift corpuscular rays through matter. Ann Phys 5(3):325-400

13. Bethe HA (1953) Moliere theory of multiple scattering. Phys Rev 89(6):1256

14. Moliere G (1948) Theorie der streuung schneller geladener teilchen-ii mehrfachstreuung und vielfachstreuung. Zeitschrift fur Naturforschung Sect A-A J Phys Sci 3(2):78

15. Henley EM, Garcia A (2007) Subatomic physics, vol 35. World Scientific

16. King NSP, Ables E, Adams K, Alrick KR et al (1999) An 800-MeV proton radiography facility for dynamic experiments. Nucl Instrum Methods Phys Res Sect A-Accel Spectrom Detect Assoc Equip 424(1):84-91

17. King NSP, Baker S, Jaramillo S, Kwiatkowski K et al (2003) Imaging detector systems for soft X-ray and proton radiography. Proc Soc Photo Opt Instrum Eng (SPIE) Int Congr High Speed Photogr Photon 4948:610-615

18. Merrill FE, Campos E, Espinoza C, Hogan G, Hollander B, Lopez J, Mariam FG, Morley D, Morris CL, Murray M (2011) Magnifying lens for $800 \mathrm{MeV}$ proton radiography. Rev Sci Instrum 82:103709

19. Mottershead CT, Zumbro JD (1998) Magnetic optics for proton radiography. In: Comyn $M$ (ed) 17th Particle Accelerator Conference. IEEE, Vancouver, pp 1397-1399

20. Beringer J, JF Arguin, RM Barnett, K Copic et al (2012) Review of particle physics. Phys Rev D 86(1)

21. Nakamura K, Hagiwara K, Hikasa K, Murayama H et al (2010) Review of particle physics. J Phys G: Nucl Part Phys 37(7A): 075021

22. Rigg A, Schwartz CL, Hixson RS, Hogan GE, Kwiatkowski KK, Mariam FG, Marr-Lyon M, Merrill FE, Morris CL, Rightly P, Saunders A, Tupa D (2008) Proton radiography and accurate density measurements: a window into shock wave processes. Phys Rev B 77(22)

23. Mottershead T, Barlow D, Blind B, Hogan G, Jason A, Merrill F, Morley K, Morris C, Saunders A, Valdiviez R (2003) Design and operation of a proton microscope for radiography at $800 \mathrm{MeV}$. IEEE

24. Danly CR, Merrill FE, Barlow D, Mariam FG (2014) Nonuniform radiation damage in permanent magnet quadrupoles. Rev Sci Instrum 85(8):083305

25. Terrones G, Burkett M, Morris C (2011) Burn front and reflected shock wave visualization in an inertially confined detonation of high explosive. Bull Am Phys Soc 56

26. Terrones G, Burkett MW, Morris CL (2012) Burn front and reflected shock wave visualization in an inertially confined detonation of high explosive. In: Shock Compression of Condensed Matter-2011: Proceedings of the Conference of the American Physical Society Topical Group on Shock Compression of Condensed Matter. AIP Publishing

27. Buttler WT, Oró DM, Preston DL, Mikaelian KO, Cherne FJ, Hixson RS, Mariam FG, Morris C, Stone JB, Terrones G (2014) Second shock ejecta measurements with an explosively driven twoshockwave drive. J Appl Phys 116(10):103519

28. Buttler WT, Oró DM, Preston DL, Mikaelian KO, Cherne FJ, Hixson RS, Mariam FG, Morris C, Stone JB, Terrones G (2012) Unstable Richtmyer-Meshkov growth of solid and liquid metals in vacuum. J Fluid Mech 703:60-84

29. Buttler W, Stone B, Oro D, Dimonte G, Preston D, Cherne F, Germann T, Terrones G, Tupa D (2011) The study of high-speed 
surface dynamics using a pulsed proton beam. Bull Am Phys Soc 56:999-1002

30. Buttler WT, Oro DM, Preston D, Mikaelian KO, Cherne FJ, Hixson RS, Mariam FG, Morris CL, Stone JB, Terrones G (2012) The study of high-speed surface dynamics using a pulsed proton beam. In: Shock compression of condensed matter-2011: Proceedings of the Conference of the American Physical Society Topical Group on Shock Compression of Condensed Matter. AIP Publishing

31. Dimonte G, Terrones G, Cherne FJ, Germann TC, Dupont V, Kadau K, Buttler WT, Oro DM, Morris C, Preston DL (2011) Use of the Richtmyer-Meshkov instability to infer yield stress at high-energy densities. Phys Rev Lett 107(26):264502

32. Clarke A, Imhoff S, Gibbs P, Cooley J, Morris C, Merrill F, Hollander B, Mariam F, Ott T, Barker M (2013) Proton radiography peers into metal solidification. Sci Rep 3:2020

33. Gibbs PJ, Imhoff SD, Morris CL, Merrill FE, Wilde CH, Nedrow P, Mariam FG, Fezzaa K, Lee W-K, Clarke AJ (2014) Multiscale xray and proton imaging of bismuth-tin solidification. JOM 66(8): $1485-1492$

34. PCO (2014) [cited 2014 10/02]. Available from: http://www.pco. de/highspeed-cameras/

35. Morris CL, Bourke M, Byler DD, Chen CF, Hogan G, Hunter JF, Kwiatkowski K, Mariam FG, McClellan KJ, Merrill F (2013) Qualitative comparison of bremsstrahlung x-rays and $800 \mathrm{MeV}$ protons for tomography of urania fuel pellets. Rev Sci Instrum 84(2):023902

36. Tremsin AS, Vogel SC, Mocko M, Bourke MAM, Yuan V, Nelson RO, Brown DW, Feller WB (2013) Non-destructive studies of fuel pellets by neutron resonance absorption radiography and thermal neutron radiography. J Nucl Mater 440(1):633-646
37. Kwiatkowski K, Douence V, Bai Y, Nedrow P, Mariam F, Merrill F, Morris CL, Saunders A(2014) Ultra-fast high-resolution hybrid and monolithic CMOS imagers in multi-frame radiography. In: SPIE Optical Engineering+Applications. International Society for Optics and Photonics

38. Bocanegra-Bernal MH, Matovic B (2009) Dense and near-netshape fabrication of Si N ceramics 500:130-149

39. Li B, Mukasyan A, Varma A (2003) Combustion synthesis of CoCrMo orthopedic implant alloys: microstructure and properties. Mater Res Innov 7:245-252

40. Merzhanov AG (1994) Solid flame: discoveries, concepts, and horizons of cognition. Combust Sci Technol 98:307-336

41. Merzhanov AG (2004) The chemistry of self-propagating hightemperature synthesis. J Mater Chem 14:1779-1786

42. Parkin IP et al (1998) Convenient, low energy routes to hexagonal ferrites MFe12O19 $(\mathrm{M}=\mathrm{Sr}, \mathrm{Ba})$ from SHS reactions of iron, iron oxide and MO2 in air. J Mater Chem 8:573-578

43. Yeh CL, Chen CF (2007) An experimental investigation on combustion synthesis of transition metal silicides V5Si3, Nb5Si5, and Ta5Si3. J Alloys Compd 439:59-66

44. Yeh CL, Hsu CC (2005) An experimental study on Ti Si formation by combustion synthesis in self propagating mode. J Alloys Compd 395:53-58

45. Yeh CL, Wang HJ (2007) A comparative study on combustion synthesis of Ta-Si compounds. Intermetallics 15:1277-1284

46. Bernert T, Winkler B, Haussühl E, Trouw F, Vogel SC, Hurd AJ, Smilowitz L, Henson BF, Merrill FE, Morris CL (2013) In situ observation of self-propagating high temperature syntheses of Ta $\mathrm{Si}, \mathrm{Ti} \mathrm{Si}$ and $\mathrm{TiB}$ by proton and X-ray radiography. Solid State Sci 22:33-42 